\title{
ROBUST SOURCE CODING FOR IMAGES OVER VERY NOISY CHANNELS
}

\author{
Lisa M. Marvel \\ U.S. Army Research Laboratory, \\ Aberdeen Proving Ground, MD 21005 \\ marvel@arl.mil \\ Ali S. Khayrallah \\ Ericsson, Inc., \\ Research Triangle Park, NC 27709 \\ Charles G. Boncelet, Jr. \\ University of Delaware, \\ Newark, DE 19716
}

\begin{abstract}
Robust source coding provides both compression and noise mitigation without channel coding. In this paper, two methods of robust source coding are presented. The first is DPCM incorporating a nonlinear filter; the second is Predictive Trellis-Coded Quantization (PTCQ), whose prediction filter is also nonlinear. Findings show that the incorporation of the nonlinear filter provides the ability to conceal the effects of noise and minimize the propagation of error. A comparison is made between the performance of the two methods in a noisy environment. It is demonstrated that the PTCQ scheme provides a method of least distortion at low BER. However, at higher BER the DPCM scheme outperforms PTCQ. Robust source coding of images employing PTCQ or DPCM with the nonlinear prediction filter exhibits low sensitivity to bit errors and yields, visually, a better reconstructed image after transmission over very noisy channels.
\end{abstract}

\section{INTRODUCTION}

The traditional technique of image transmission requires a multistage process. The first stage is source coding, or the removal of redundancy, to compress the image for a narrow bandwidth channel. The second stage requires coding for the channel by adding redundant characters to protect the information from noise.

We pursue a method that will perform robust source coding, as a technique which provides both compression and noise mitigation without channel coding. Specifically, the utilization of two source coding schemes,

This research was funded by the Army Research Laboratory under Cooperative Agreement No. DAAL01-96-2-0002. The U.S. Government is authorized to reproduce and distribute reprints for Government purposes notwithstanding any copyright notation thereon.
PTCQ and DPCM, incorporating a linear and nonlinear filter is investigated. This research is motivated by the original paper on PTCQ applied to speech signals by Marcellin and Fischer [1] and the recent work of Khayrallah [2] and Marvel et al. [3].

Predictive coding requires an extremely simple implementation. Although the compression rate associated with this method is low in comparison to other techniques, the low complexity and real-time implementability provides an incentive for use. In addition, many methods incorporate predictive coding; as an example the JPEG algorithm utilizes DPCM to encode the DC coefficients of the discrete cosine transform.

This paper provides a description of PTCQ, evaluates the incorporation of linear and nonlinear filters, and exhibits their resiliency to noise. In addition, the affects of various PTCQ parameters, such as bit rate and the number of trellis states, on performance are discussed. Lastly, the performance of both systems is evaluated for a range of channel noise levels.

\section{PTCQ}

PTCQ as a method of image transmission achieves compression with minimal distortion and modest computational complexity. Trellis-Coded Quantization (TCQ) employs the use of an expanded set of quantization levels along with maximum likelihood decoding applying the Viterbi algorithm. This algorithm determines the path of minimal distortion through a trellis, whose structure allows for low bit rate encoding. PTCQ combines the encoding efficiency of TCQ with the compression merits of lossy predictive coding.

At each stage and state in the trellis, the prediction error is computed as the difference between the output of the prediction filter, whose input is specified by the surviving path into the state, and the current pixel 


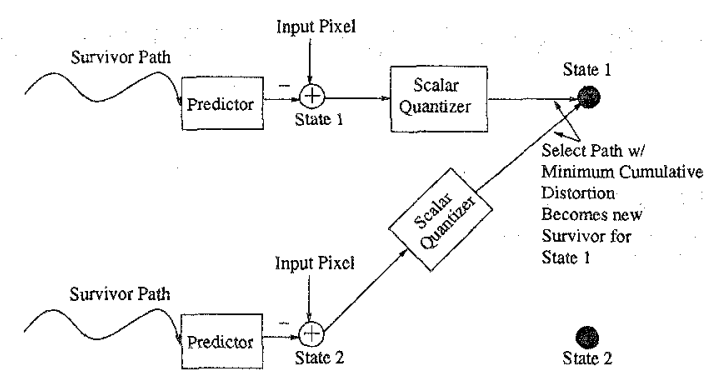

Figure 1: Abstract Diagram of PTCQ

value. This prediction error is then quantized using the partitioned scalar quantizer assigned to the particular state transition. This operation is performed for each branch entering a state. The surviving path into a state is chosen as the path entering the state with the least cumulative distortion; all other paths are discarded. The output of the PTCQ algorithm representing each pixel has two portions: the branch bit and the scalar quantization bits. The branch bit specifies which branch to take within the trellis, and the scalar quantization bit selects the quantization point within the partitioned scalar quantizer. An abstract diagram of the PTCQ algorithm is depicted in Figure 1 using a two-state trellis.

The trellises are generated using a convolutional encoder. Traditionally, the convolutional encoders utilized for trellis coding have feedback. However, encoded output from this type of trellis can result in catastrophic failure when transmitted over a noisy channel. To remedy this problem, a convolutional encoder without feedback must be used for trellis generation [4]. Fortunately, for every convolutional encoder with feedback, there exists a feedback-free encoder, for which any given input bit can affect no more that $1+\log _{2}(N)$ outputs, where $N$ is defined as the number of trellis states [5]. A feedback-free convolutional encoder produces a similar trellis with the branch bits labeled differently. This construction bounds the diversion from the correct trellis path caused by a bit error and allows the intended path to be recovered.

\section{PREDICTION FILTERS}

The prediction filters used for robust coding are causal; therefore, image reconstruction incurs no delay. A conventional prediction filter used in predictive coding is the optimal linear filter. This filter is a linear combination of previous pixels. The coefficients for this filter depend only on the autocorrelation of pixels in the original image.

The nonlinear filter applied here is a special case of the Ll-filter [6]. The nonlinear filter coefficients are selected based on the ranking of elements within a fivepoint window. Filter coefficients for the minimum and maximum values within the filter window are set to zero, while the remaining coefficients are specified by the autocorrelation of pixels within the original image as with the optimal linear filter.

\section{NOISY CHANNEL PERFORMANCE}

Figures 2 and 3 display the performance of the optimal linear filter within the PTCQ and DPCM schemes, respectively, in a noisy environment with a BER of $10^{-2.5}$. Figures 4 and 5 demonstrate the effectiveness of the nonlinear filter within the two schemes, operating in the same noisy environment. The error propagation is very evident in the linear filter implementation. However, the nonlinear filter provides the nonlinearity necessary to minimize the propagation of error and result in a reconstructed image that is visually superior.

In order to quantize the prediction error, DPCM utilizes an optimal scalar quantizer where PTCQ employs the method of TCQ that combines optimal scalar quantization with the principles of Trellis-Coded Modulation. It is well known that TCQ outperforms optimal scalar quantization [1]. Given this fact, it is logical to suspect that PTCQ will achieve a lower distortion than DPCM.

By implementing a four-state PTCQ scheme operating at $3 \mathrm{bpp}$ and comparing it to DPCM operating at the same rate, we found this to be true for low BER. Linear (l)-PTCQ outperforms $l$-DPCM by $3.5 \mathrm{~dB}$ PSNR and nonlinear ( $\mathrm{L} l$ )-PTCQ exceeds $\mathrm{L} l$-DPCM by $3.32 \mathrm{~dB}$ at low BER. However, at BER above $10^{-2.5}$, the method of least distortion becomes the DPCM scheme. l-DPCM performs $1.22-2 \mathrm{~dB}$ PSNR better than $l$-PTCQ, and $\mathrm{L} l$-DPCM exceeds $\mathrm{L} l$-PTCQ in performance by $.6-1.27 \mathrm{~dB}$ PSNR. This performance is depicted for both the linear filter and the nonlinear filter implementations, as indicated in Figures 6 and 7 , respectively.

PTCQ parameters also have an affect on the performance of the algorithm in the presence of noise. The amount of propagation from a single error in the branch bit is upper bounded by $1+\log _{2}(N)$, where $N$ is the number of trellis states. Therefore, a diversion from the correct path caused by a bit error would affect more output symbols as the number of trellis states is increased. The best performance over very noisy channels was achieved with two-state PTCQ system operating at two to three bpp. This configuration outperformed other PTCQ configurations with a greater number of states and/or increased bit rate. 


\section{CONCLUSIONS}

The phenomenon of degraded performance of $\mathrm{PTCQ}$ at increased BER is attributed to the construction of PTCQ, If an error is made within the branch bit, it can affect no more than $1+\log _{2}(N)$ inputs where $N$ is the number of trellis states. However, if a bit error occurs in the scalar quantization bits, the result of this error is no more severe than a scalar quantization error in the DPCM scheme. The property that allows PTCQ to exceed DPCM at low BER, namely the trellis structure, provides a hindrance when the BER is increased.

We have presented two methods of robust source coding which provide compression and error resiliency without adding a channel coding stage. These simple algorithms are implementable in near real-time. For both systems the incorporation of the nonlinear filter limits the propagation of error and provides noise mitigation in the presence of channel errors. At low BER PTCQ outperforms DPCM by approximately $3.3-3.5$ dB PSNR. However, at BER greater than $10^{-2.5}$, the DPCM scheme should be utilized to achieve a gain of .6 - $2 \mathrm{~dB}$ PSNR over PTCQ.

\section{REFERENCES}

[1] M. W. Marcellin and T. R. Fischer. Predictive trellis coded quantization of speech. IEEE Transactions on Acoustics, Speech and Signal Processing, $38(1): 46-55$, January 1990.

[2] A. S. Khayrallah. Nonlinear filters in joint source channel coding of images. Presented at the IEEE International Symposium on Information Theory, Whistler, CA, September 1995.

[3] L. M. Marvel, A. S. Khayrallah, and C. G. Boncelet Jr. Robust source coding of images for tactical channels. Presented at the 20th Army Science Conference, Norfolk, VA, June 1996.

[4] M. Wang and T. R. Fischer. Trellis-coded quantization designed for noisy channels. IEEE Transactions on Information Theory, 40(6):1792-1802, Novernber 1994.

[5] M. W. Marcellin and T. R. Fischer. Trellis coded quantization of memoryless and Gauss-Markov sources. IEEE Transactions on Communications, 38(1):82-93, January 1990.

[6] F. Palmieri and C. G. Boncelet Jr. Ll-filters - a new class of order statistic filters. IEEE Transactions on Acoustics, Speech, and Signal Processing, 37(5):691-701, May 1989.
The views and conclusions contained in this document are those of the authors and should not be interpreted as representing the official policies, either expressed or implied, of the Army Research Laboratory or the U.S. Government.

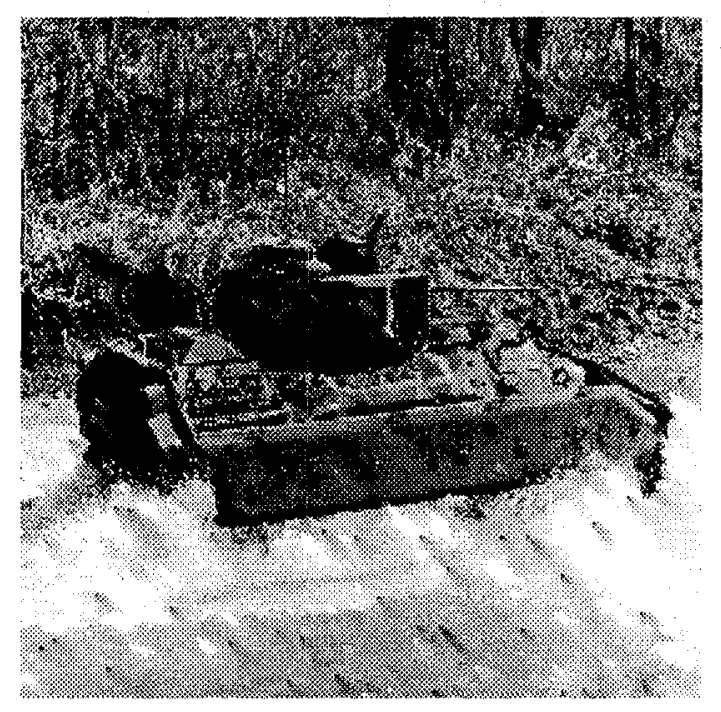

Figure 2: Linear PTCQ - Noisy Channel

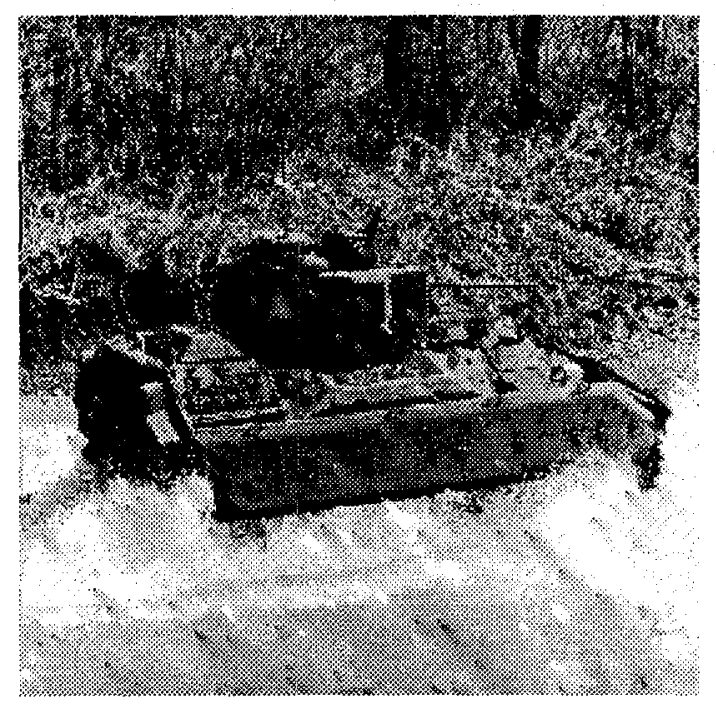

Figure 3: Linear DPCM - Noisy Channel 


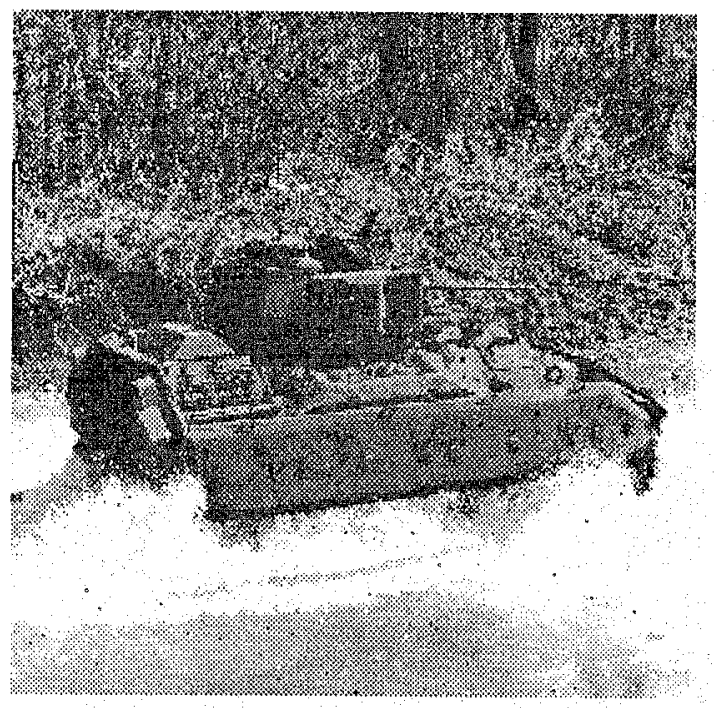

Figure 4. Nonlinear PTCQ - Noisy Channel

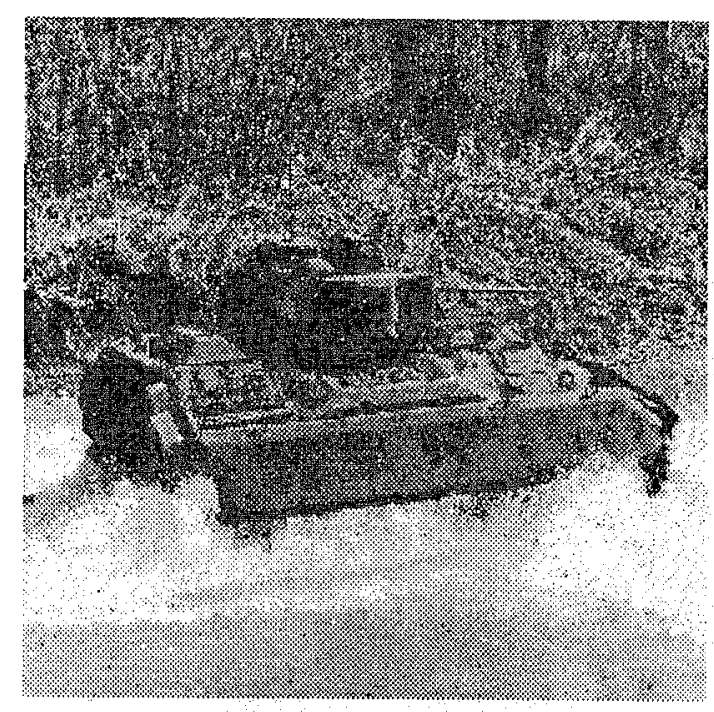

Figure 5: Nonlinear DPCM - Noisy Channel

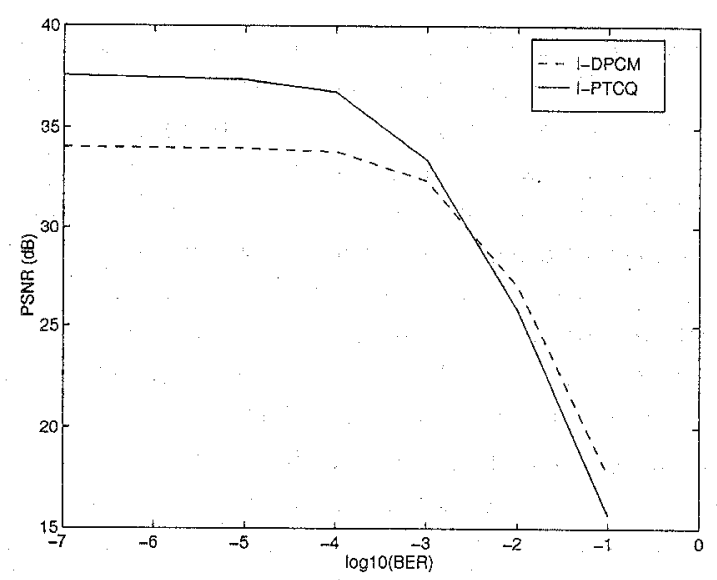

Figure 6: Performance of l-Filter Implementation

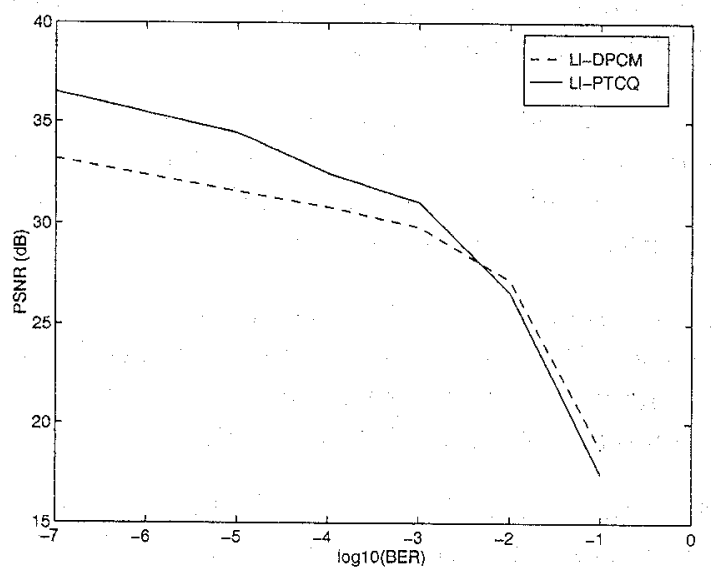

Figure 7: Performance of $\mathrm{L} l$-Filter Implementation 